\title{
Ethnic Identity, Drinking Motives, and Alcohol Consequences Among Alaska Native and Non-Native College Students
}

\section{Authors: Monica C. Skewes \& Arthur W. Blume}

This is an Accepted Manuscript of an article published by Taylor \& Francis in Journal of Ethnicity in Substance Abuse in December 2014, available online:

http://www.tandfonline.com/10.1080/15332640.2014.958641

Skewes, Monica C., and Arthur W. Blume. "Ethnic Identity, Drinking Motives, and Alcohol Consequences Among Alaska Native and Non-Native College Students." Journal of Ethnicity in Substance Abuse 14, no. 1 (December 20, 2014): 12-28. doi: $10.1080 / 15332640.2014 .958641$.

Made available through Montana State University's $\underline{\text { ScholarWorks }}$ scholarworks. montana.edu 
Running Head: DRINKING MOTIVES AND ETHNIC IDENTITY

Ethnic Identity, Drinking Motives, and Alcohol Consequences Among Alaska Native and NonNative College Students

\author{
Monica C. Skewes ${ }^{1}$ and Arthur W. Blume ${ }^{2}$ \\ ${ }^{1}$ Department of Psychology, Montana State University \\ ${ }^{2}$ Department of Psychology, Washington State University Vancouver
}

Correspondence concerning this manuscript should be addressed to Monica C. Skewes, Ph.D., Department of Psychology, 325 Traphagen Hall, Montana State University, Bozeman, MT 59715.

Email: monica.skewes@montana.edu 


\begin{abstract}
This research involves the examination of drinking motives, alcohol consequences, and ethnic identity in a sample of Native and non-Native college student drinkers in Alaska. Although more Alaska Native students are abstinent from alcohol than any other ethnic group, Native students who do drink experience greater alcohol consequences and dependence symptoms. Therefore, we attempted to examine the influence of ethnic identity on alcohol consequences in a diverse sample of Native and non-Native students in Alaska. Findings showed that drinking motives as measured by the Drinking Motives Questionnaire (social, coping, enhancement, and conformity) significantly predicted alcohol consequences after controlling for frequency of monthly binge drinking. Also, after controlling for depression, binge drinking, and drinking motives, one aspect of ethnic identity (Affirmation, Belonging, and Commitment) was significantly negatively related to alcohol consequences, whereas another aspect of ethnic identity (Ethnic Identity Search) was not. Taken together, these findings suggest that interventions for college student alcohol misuse that target Native students should be culturally grounded and focused on enhancing Affirmation, Belonging, and Commitment to one's ethnic heritage, and also should address drinking motives, especially drinking to cope, as a way to reduce alcohol related harm.
\end{abstract}


Excessive alcohol use among college students is both common and dangerous. It is estimated that nearly half of U.S. college students report engaging in binge drinking (defined as consuming five or more standard drinks in one sitting for men and four or more standard drinks for women) within the past two weeks (McNally \& Palfai, 2001; Wechsler \& Nelson, 2001). The negative consequences associated with binge drinking in this population include health problems, legal problems, academic problems, negative social consequences, unwanted or unsafe sexual experiences, violence and aggression, and accidents/personal injuries (Alexander \& Bowen, 2004; Blume, Schmaling \& Marlatt, 2003; Park, 2004; O’Malley \& Johnston, 2002). It has been estimated that $31 \%$ of college students have symptoms consistent with a diagnosis of alcohol abuse and 6\% meet criteria for alcohol dependence (Knight et al., 2002). Despite the potential harms, excessive alcohol consumption remains a common occurrence among college students and a national public health concern (Courtney \& Polich, 2009; Hingson, Heeren, Winter, \& Wechsler, 2005).

Higher rates of alcohol dependence and associated consequences have been documented among both Native and non-Native Alaskans, with $14 \%$ of all Alaskan adults suffering from alcohol abuse or dependence (O’Malley et al., 2008). While American Indians and Alaska Natives are more likely than any other ethnic group to be abstinent from alcohol, Native people who do drink consume greater quantities of alcohol and are more likely to meet DSM criteria for dependence (Beauvais, 1992; Beals et al., 2005; Whitesell, Beals, Big Crow, Mitchell, \& Knovins, 2012). Although there is insufficient research to draw conclusions about ethnic disparities in alcohol use and consequences among Alaska Native college students, previous large-scale epidemiological surveys have found consistently that Native adolescents report greater alcohol consumption than White adolescents (Blum, Harmon, Harris, Bergeisen, \& 
Resnick, 1992; Beauvais, 1992; Beauvais, 1996; Wallace et al., 2002). This disparity may contribute to the low rates of college graduation among Alaska Natives (NCES, 2012). Understanding reasons for drinking among Alaska Native college students may facilitate the development or adaptation of effective and culturally compatible intervention strategies.

\section{Drinking Motives}

Motivational models of alcohol use posit that people drink alcohol for various reasons, and these reasons or motives for drinking are uniquely related to both antecedents and consequences of drinking behavior (Cooper, 1994; Cooper, Frone, Russell, \& Mudar, 1995). Motives are conceptualized as a distinct construct from expectancies, or the expected consequences of drinking behavior. One might expect a certain outcome from drinking (e.g., hangover) that may not be a reason to consume alcohol. However, some expectancies and motives may be related to one another-for example, drinking to have fun or to relieve negative affect may be both motives for drinking and expected outcomes. Evidence exists that drinking motives are important psychological factors in predicting drinking patterns and related problems among adolescents and young adults from various ethnic backgrounds and nationalities (Cooper, 1994; Kuntsche, Stewart, \& Cooper, 2008; Bernstein, Graczyk, Lawrence, Bernstein, \& Strunin, 2011).

Cooper (1994) examined four motives for alcohol use based on a motivational model developed by Cox and Klinger (1988), including social, coping, enhancement, and conformity motives. Social motives are associated with drinking to obtain social rewards, such as facilitating social interactions and bonds with others. Coping motives result in drinking to manage negative mood states. Enhancement motives result in drinking to enhance positive mood and to experience pleasure. Conformity motives result in drinking to avoid social sanctions such as 
rejection or disapproval. These motives can be seen as attempts to feel good (i.e., seek positive reinforcement via social or enhancement motives) or as attempts to avoid feeling bad (i.e., seek negative reinforcement by relieving or avoiding negative affect or social consequences via coping or conformity motives).

A measure developed from this model, now known as the Drinking Motives Questionnaire-Revised (DMQ-R; Cooper, 1994), was found to be valid and reliable in a study of 1,243 Black and White adolescents, and the four-factor structure was invariant across gender, age, and race (Cooper, 1994). Also, internal consistency estimates were found to be essentially equivalent across demographic subgroups. A cross-national validation study of the DMQ-R in Switzerland, Canada, and the U.S. found this measure to be valid and reliable across cultures with good internal consistency (coefficient alpha $\geq .82$ for each subscale) and invariant factor structure (Kuntsche, Stewart, \& Cooper, 2008).

Drinking motives as measured by the DMQ-R have been shown to predict drinking behavior and alcohol outcomes. In a series of multiple regression analyses using these four motives as predictors of drinking behavior, the set of motives significantly predicted both quantity and frequency of drinking, accounting for $14 \%$ to $20 \%$ of the variance (Cooper, 1994). However, different types of motives have been shown to have unique relationships with drinking antecedents, patterns, contexts, and consequences (Cooper et al., 1995; Carey \& Correia, 1997). Drinking in response to various motives may be conceptualized as behaviors or patterns of alcohol use that are qualitatively distinct from one another and may warrant different intervention approaches.

Social motives are those frequently endorsed by drinkers and associated with drinking in social settings (e.g., at parties) and with frequent, nonproblematic drinking (Cooper, Russell, 
Skinner, Frone, \& Mudar, 1992; Cooper, 1994). The majority of young people who drink do so for social reasons, and social motives are associated with moderate as opposed to excessive drinking (Kuntsche et al., 2005; LaBrie, Hummer, \& Pedersen, 2007). Social motives also are negatively associated with drinking alone (Cooper, 1994), a style of drinking shown to be related to depression, suicidal ideation, alcohol problems, and symptoms of alcohol dependence (Gonzalez, 2012; Gonzalez \& Skewes, 2013).

Drinking to cope, compared with other motives, predicts indirect (via increased consumption) and direct effects on alcohol problems (Cooper, 1994). That is, coping motives are consistently associated with more severe alcohol consequences even after controlling for alcohol consumption (Cooper et al., 1995, Kuntsche, 2007) or high-risk drinking (Carey \& Correia, 1997). People who drink to cope may do so because they have not developed other more adaptive coping skills, and using alcohol as a coping mechanism may further erode or delay the development of alternative coping skills, potentially contributing to worse alcohol problems (Cooper et al., 1995). Of the four motives discussed, coping has been consistently shown to be the strongest predictor of alcohol consequences, whereas enhancement has been the strongest predictor of heavy drinking.

Enhancement motives are the second most frequently endorsed drinking motives, after social motives, among young adults (Kuntsche et al., 2005). They have been shown to predict heavy drinking, but affect alcohol problems indirectly through increased alcohol consumption (Cooper et al., 1995; Kuntsche et al., 2005). When controlling for drinks per drinking day, enhancement motives no longer significantly predicted alcohol problems, whereas coping and conformity motives were significant predictors of alcohol problems even after controlling for alcohol use (Cooper, 1994; Kuntsche, 2007). 
Finally, conformity motives are generally not associated with quantity of alcohol consumed or frequency of drinking, but directly predict greater alcohol problems (Cooper, 1994). This motive is the one least frequently endorsed, with more adolescents than young adults reporting drinking for conformity reasons. Relationships between drinking motives, alcohol consumption, and alcohol problems have been shown to be stable across ethnic groups, genders, and age groups (Cooper, 1994). These findings suggest that people whose drinking is negatively reinforced (i.e., coping or conformity motives) are at greater risk for alcohol problems than people whose drinking is positively reinforced (i.e., social or enhancement motives).

Extensions of this research with college students have found that drinking motives significantly predicted alcohol problems after controlling for maximum number of drinks consumed on a single day and frequency of drinking to intoxication in the last month, and that negative reinforcement motives had stronger associations with problems than positive reinforcement motives (Carey \& Correia, 1997). In a cross-cultural examination, Neff (1997) found increased quantity and frequency of binge drinking among White, African American, and Latino college students associated with escape motives, which may be conceptualized within the present framework as akin to coping motives. Another study of U.S. college students found that coping but not enhancement or social motives significantly predicted alcohol problems (Kassel, Jackson, \& Unrod, 2000). In spite of evidence that drinking motives may be important to understand and intervene upon aversive drinking consequences, the relationship between drinking motives and alcohol consequences has not been examined among Native college students. 


\section{Ethnic Identity and Alcohol Problems}

There is reason to believe that ethnic identity may be protective against alcohol use and problems. Research has found that Mexican American women who are more acculturated to the majority culture use more alcohol, and those who are less acculturated (i.e., more enculturated to traditional Mexican culture) use less alcohol (Caetano \& Clark, 2003; Oetting, Donnermeyer, Trimble, \& Beauvais, 1998). Other studies found lower rates of alcohol and marijuana use among African American young adults with higher levels of ethnic identity (Pugh \& Bry, 2007). Marsiglia, Kulis, and Hecht (2001) found that ethnic minority youth with a strong sense of ethnic pride reported less drug use, whereas White youth with a strong sense of ethnic pride reported more drug use. Among American Indians, youth with a stronger sense of ethnic pride were more likely to adhere to anti-drug norms regarding alcohol, tobacco, and marijuana, and they also reported having greater self-efficacy to refuse substances if offered by their peers (Kulis, Napoli, \& Marsiglia, 2002).

On the other hand, findings regarding the link between ethnic identity and substance use among ethnic minorities have been inconsistent. In a study of culturally diverse Australian adolescents, there were few statistically significant associations between ethnic identity and substance use, and most of the protective effects for ethnic identity were evidenced for nonIndigenous, White respondents (Gazis, Connor, \& Ho, 2010). Similarly, in a study of youth in the U.S. Southwest, researchers found that the protective effects of ethnic identity were stronger for White than for minority respondents (Marsiglia, Kulis, Hecht, \& Stills, 2004). One study found that identification with the "Indian way of life" predicted actually greater alcohol problems (Hawkins, 2002), while another study found no association between ethnic identity and substance use (Bates, Beauvais, \& Trimble, 1997). While many researchers continue to believe 
that ethnic identity may indeed protect ethnic minority people from alcohol use and problems, the mixed findings in the literature indicate that these associations are not yet well understood. Furthermore, it is not clear what dimension of ethnic identity may be protective from alcohol use and consequences among ethnic minority college students.

\section{Present Study}

Because of the high rates of binge drinking on college campuses and the increased risk of alcohol problems among Native peoples, not to mention the poor rates of graduation from college among Native students, it is important to examine potential protective factors such as ethnic identity. It also is important to examine potential risk factors for heavy drinking and alcohol problems, such as drinking motives, which have not been previously studied among Native students. The present study sought to compare alcohol problems between Native and nonNative college students while controlling for alcohol consumption. We hypothesized that Native students would experience greater alcohol-related consequences than non-Native students. We also sought to examine the potential effects of different drinking motives on alcohol consequences, hypothesizing that drinking to cope would be more strongly and significantly associated with negative consequences than drinking for social, enhancement, or conformity reasons. Finally, we examined the effects of two dimensions of ethnic identity - ethnic identity achievement and affirmation, belonging, and commitment — on alcohol problems after controlling for ethnicity, binge alcohol consumption, and drinking motives. We hypothesized that ethnic identity would be predictive of fewer alcohol problems after controlling for binge alcohol consumption and motives. 
Method

\section{Participants}

The participant pool included 298 undergraduate college students enrolled at a university in the northern US. Respondents who reported no alcohol consequences within the past 12 months were excluded from the following analyses, resulting in a study sample of 205 students aged from $18-52(M=22.80, S D=5.75)$. The majority of students were emerging adults $(81.9 \%$ were between 18 and 25 years of age) and freshmen $(n=67,32.7 \%)$, with the remainder of the sample evenly distributed between sophomores, juniors, and seniors. Regarding ethnicity, 68.3\% $(n=140)$ students self-identified as White, $14.1 \%(n=29)$ identified as Alaska Native or American Indian, and 17.6\% $(n=36)$ were other ethnic minorities including African American, Latino, and Asian American.

\section{Measures}

Alcohol Use. The Alcohol Use Disorders Identification Test (AUDIT; Saunders, Aasland, Babor, De La Fuente, \& Grant, 1993) is an 8-item self-report screening measure developed by the World Health Organization as a screening tool for hazardous drinking. The first three questions of the AUDIT (AUDIT-C) have been used effectively to assess problematic alcohol consumption (Bush, Kivlahan, McDonnell, Fihn, \& Bradley, 1998). Estimations of the number of binge drinking episodes per month and the number of standard drinks consumed per typical month were calculated using the AUDIT-C.

Alcohol problems. The Young Adult Alcohol Consequences Questionnaire (YAACQ; Read, Kahler, Strong, \& Colder, 2006) is a 48-item self-report inventory of problems commonly associated with alcohol use among college students. Each item is scored as present (1) or absent 
(0) within the past 12 months. Item responses are summed to yield a total score from $0-48$, with higher scores indicative of greater alcohol problems, which was used in subsequent analyses.

Drinking motives. The Drinking Motives Questionnaire-Revised (DMQ-R; Cooper, 1994) is a 20-item measure of drinking motives, including four 5-item subscales assessing drinking for social reasons (e.g., to bond with others), drinking to cope with negative affect (e.g., to forget one's problems), drinking for enhancement reasons (e.g., to increase enjoyment or have fun), and drinking for conformity reasons (e.g., drinking to fit in). The measurement items of the questionnaire ask respondents to indicate how frequently they drink in response to these motives on a scale of 1 (almost never) to 4 (almost always). Possible range for each subscale (Social, Coping, Enhancement, Conformity) was 5-20, with higher scores indicating greater self-reported drinking in response to a particular motive. Total scores for each scale were used in subsequent analyses.

Depression. The Beck Depression Inventory-II (BDI-II; Beck, Steer, \& Brown, 1996) is a 21-item self-report measure of depressive symptoms experienced during the past two weeks. Items are scored from 0 to 3, with higher scores indicating greater depressive symptoms. A total score (0-63) derived by summing the item scores was used in subsequent analyses.

Ethnic identity. The Multigroup Ethnic Identity Questionnaire (MEIM; Phinney \& Ong, 2007 ) is a 15 -item measure of ethnic identity that is not specific to any one ethnicity. Respondents are asked to write-in the ethnicity with which they most identify at the top of the page, and the 12 subsequent questions refer to that self-identified group. Items 13, 14, and 15 are not included in the total or subscale scores, but are used for qualitative ethnic identification and categorization. Items 1 through 12 are rated from 1 (strongly disagree) to 4 (strongly agree). Two subscale scores representing ethnic identity search (EIS) and affirmation, belonging, and 
commitment $(\mathrm{ABC})$ are calculated by taking the mean of the items that comprise each subscale. EIS is conceptualized as a developmental and cognitive factor; a sample item is "I have spent time trying to find out more about my own ethnic group, such as its history, traditions, and customs." ABC is conceptualized as an affective factor; a sample item is "I have a clear sense of my ethnic background and what it means for me." Both the MEIM EIS and ABC subscale scores can range from 1-4 and were used for subsequent analyses in the study.

\section{Procedure}

Participants were recruited via in-class announcements and received extra course credit for their time. They provided informed consent and then completed packets of paper-and-pencil measures in small groups. Students who had not experienced consequences of drinking during the previous year were excluded from the study, yielding a study sample of 205 students. Each participant was given a manila envelope with the survey and instructed to place the completed survey into the envelope and then to place the envelope into a large box filled with other completed surveys. This procedure was intended to enhance participants' confidence that their responses would not be identifiable or linked back to them in any way, thereby improving validity of self-reports. All methods and materials were approved by the university's Institutional Review Board.

Analyses

First, independent samples t-tests were performed to test differences in mean scores for all study variables between Native and non-Native students. Ethnicity was determined by selfreport, and students from Alaska Native and/or other American Indian tribal groups were classified as "Native," with all others defined as non-Native for the purposes of study analyses. The decision to compare Native with non-Native students was made deliberately in consideration 
of cultural stereotypes toward Native people who drink. Although all ethnic minority groups suffer from discrimination, there is a strong stereotype in the U.S. about Native people who drink alcohol that is unique to indigenous people (Mail, 2002). We believe that the effects of this stereotype, also known as the "firewater myth" (LaMarr, 2003), may influence Native people who drink differently than other ethnic minorities due to the stigma it creates. A series of hierarchical multiple regression analyses was conducted to assess the association of ethnic identity with alcohol problems, controlling for patterns of alcohol use, depression, and drinking motives. We hypothesized that greater ethnic identity may be protective for alcohol problems after controlling for alcohol use, depression, and drinking motives.

Four regression analyses were conducted, each controlling for a different subscale from the DMQ-R (i.e., Social, Coping, Enhancement, and Conformity). Age and gender were entered in the first step; DMQ-R subscale scores, BDI-II scores, and number of binge episodes per month were entered in the second step; ethnicity (Native or non-Native) was entered in the third step; and both MEIM subscale scores (EIS and ABC) were entered in the fourth step. Total YAACQ scores served as the dependent variable in each model.

\section{Results}

See Table 1 for scale means and standard deviations. Independent samples $t$-tests showed that Native students reported significantly greater alcohol consequences and greater ethnic identity than non-Native students as evidenced by significantly higher YAACQ scores, MEIMEIS scores, and MEIM-ABC scores (see Table 1). However, Native and non-Native students had statistically equivalent scores on the DMQ-R subscales, the AUDIT-C, and the BDI-II. In all four regression models predicting alcohol consequences from the set of predictor variables specified above, Native students reported significantly greater alcohol consequences than non- 
Native students after controlling for the number of binge drinking episodes per month (see Tables 2-5).

When social motives were included in the model (Full model $R^{2}=.36 ; F(8,178)=12.70$; $p<.001$; see Table 2), greater alcohol consequences were significantly predicted by higher BDIII scores, greater frequency of binge episodes, greater social drinking motives, and being Native. The MEIM EIS subscale scores did not predict alcohol consequences, but ABC subscale scores did. Students who reported greater Affirmation, Belonging, and Commitment to their ethnic group reported significantly fewer alcohol problems.

When drinking to cope with negative affect was included in the model (Full model $R^{2}=$ $.38 ; F(8,178)=15.44 ; p<.001$; see Table 3$)$, greater alcohol consequences similarly were predicted by greater frequency of binge drinking episodes, greater coping motives, and being Native. However, BDI-II scores did not significantly predict alcohol consequences in this model, likely because of the shared variance between depression and drinking to cope. As in the previous regression model, the MEIM-EIS subscale was not a significant predictor of alcohol consequences, but $\mathrm{ABC}$ subscale scores again appeared protective.

For the model including enhancement motives (Full model $R^{2}=.32 ; F(8,176)=12.04 ; p$ $<.001$; see Table 4), significant predictors of greater alcohol problems again included higher BDI-II scores, greater frequency of binge episodes, greater drinking for enhancement reasons, and being Native. The EIS subscale of the MEIM was non-significant, but higher ABC subscale scores predicted lower alcohol consequences. A similar pattern of results was found for the model that included conformity motives, with greater BDI-II scores, greater frequency of binge episodes, and being Native emerging as significant predictors of greater alcohol problems (Full model $R^{2}=.31 ; F(8,178)=11.34 ; p<.001 ;$ see Table 5$)$. The conformity drinking motive, 
however, was not significant. Ethnic identity functioned in the same way as in previous models, with EIS remaining non-significant and greater $\mathrm{ABC}$ subscale scores significantly predicting lesser alcohol problems.

\section{Discussion}

These findings suggest that the greater alcohol consequences reported by Native students may not entirely be accounted for by alcohol consumption, depression, or different drinking motives. Indeed, in all four regression models predicting alcohol consequences from the set of predictor variables including DMQ subscale scores, Native students reported significantly greater consequences than non-Native students after controlling for the number of binge drinking episodes per month (see Tables 2-5). These results are in contrast to Cooper's (1994) research showing that social and conformity motives only had indirect effects on alcohol consequences whereas coping and enhancement motives had both direct and indirect effects. It is possible that examining consequences in the context of binge episodes per month rather than typical drinks per month accounted for the difference in findings.

One potentially important consideration in understanding differences between Native and non-Native students' self-reported alcohol consequences involves the possibility of misperceived drinking norms. It is well established that college students commonly overestimate the quantity of alcohol typically consumed by their peers (Perkins \& Berkowitz, 1986; Berkowitz \& Perkins, 1987; Perkins, Haines, \& Rice, 2005) and that correcting this misperception leads to reductions in alcohol use and consequences (Neighbors, Larimer, \& Lewis, 2004; Carey, Scott-Sheldon, Carey, \& DeMartini, 2007; Haines \& Spear, 2010). Although unstudied in Alaska Native college students, it could be that misperceptions of peers' alcohol use influence Native students' drinking behavior, resulting in more binge drinking and alcohol consequences. This warrants further examination, however, especially considering the relatively high rates of alcohol abstinence 
among Native students and given the finding that Native students reported greater consequences even after controlling for alcohol consumption.

It also is possible that other factors such as stereotyping and prejudice contribute to the direct effects of drinking motives on alcohol consequences among Native students. For example, the measure of consequences included items associated with negative self-image due to drinking (e.g., "I have felt badly about myself because of my drinking," and "I have felt guilty about my drinking”). Native students who drink may experience negative self-image when drinking any alcohol at all due to social and cultural stereotypes associated with "the drunken Indian" (Mail, 2002; LaMarr, 2003) that results in social stigma.

It is interesting that the two subscales of the MEIM asserted effects on alcohol consequences in the same direction regardless of drinking motive. EIS was unrelated to alcohol consequences in all models, whereas ABC was significantly negatively associated with alcoholrelated consequences in all models, regardless of the motive for drinking. This suggests that a sense of belonging and positive regard toward one's ethnic group may serve as a protective factor for Native students. Exploring one's ethnic identity was not significantly associated with alcohol-related consequences, suggesting that Native students who are searching to understand their identity do not experience similar protection as those who are already committed to their Native identities. More research is needed to understand the mechanisms by which this aspect of ethnic identity protects Native students.

Taken together, these findings suggest that interventions aimed at reducing alcohol related harm among Native students should address drinking motives explicitly, possibly in conjunction with personalized normative feedback if research suggests Native students are misperceiving social norms for drinking. Although all four motives were significant predictors of 
consequences after controlling for frequency of binge drinking, the standardized regression coefficient for the coping motive $(\beta=.35)$ indicates that drinking to cope had a stronger effect on alcohol consequences than social $(\beta=.23)$, enhancement $(\beta=.20)$, or conformity $(\beta=.11)$ motives. Harm reduction strategies might include cognitive behavioral skills training to teach alternative ways of coping with distress and distress tolerance skills. Other intervention strategies supported by this research include efforts to strengthen affirmation, belonging, and commitment to one's cultural group. When students feel that they are connected to their heritage and engage in activities that strengthen their commitment to their culture, they may be protected from alcohol consequences regardless of whether or not they drink. Enhancing this component of ethnic identity in and of itself may prove to be a useful harm reduction strategy among Native students who drink. 


\section{References}

Alexander, E. N., \& Bowen, A. M. (2004). Excessive drinking in college: Behavioral outcome, not binge, as a basis for prevention. Addictive Behaviors, 29, 1199-1205.

Bates, S. C., Beauvais, F., \& Trimble, J. E. (1997). American Indian adolescent involvement and ethnic identification. Substance Use \& Misuse, 32, 2013-2031.

Beauvais, F. (1992) Trends in Indian adolescent drug and alcohol use. American Indian and Alaska Native Mental Health Research, 5, 1-12.

Beauvais, F. (1996). Trends in drug use among American Indian students and dropouts, 1975 to 1994. American Journal of Public Health, 86, 1594-1598.

Beals, J., Manson, S. M., Whitesell, N. R., Spicer, P., Novins, D. K., \& Mitchell, C. M. (2005). Prevalence of DSM-IV disorders and attendant help-seeking in 2 American Indian reservation populations: Mental health disparities in a national context. The American Journal of Psychiatry, 162, 1723-1732.

Beck, A. T., Steer, R. A., Brown, G. K. (1996). Manual for the Beck Depression Inventory-II. San Antonio, TX: Psychological Corporation.

Berkowitz, A. D., \& Perkins, H. W. (1987). Current issues in effective alcohol education programming. In Sherwood, J. S. (Ed.) Alcohol Policies and Practices on College and University Campuses (pp. 69-85). Washington, DC: National Association of Student Personnel Administrators.

Bernstein, J., Graczyk, A., Lawrence, D., Bernstein, E., \& Strunin, L. (2011). Determinants of drinking trajectories among minority youth and young adults: The interaction of risk and resilience. Youth \& Society, 43, 1199-1219. 
Blum, R. W., Harmon, B., Harris, L., Bergeisen, L., \& Resnick, M. D. (1992). American IndianAlaska Native youth health. Journal of the American Medical Association, 267, 16371644.

Blume, A. W., Schmaling, K. B., \& Marlatt, A. G. (2003). Predictors of change in binge drinking over a 3-month period. Addictive Behaviors, 28, 1007-1012.

Bush, K. R., Kivlahan, D. R., McDonell, M. B., Fihn, S. D., \& Bradley, K. A. (1998). The AUDIT alcohol consumption questions (AUDIT-C): An effective brief screening test for problem drinking. Archives of Internal Medicine, 158, 1789-1795.

Caetano, R., \& Clark, C. L. (2003). Acculturation, alcohol consumption, smoking, and drug use among Hispanics. In K. M. Chun, P. Balls Organista, \& G. Marin (Eds.), Acculturation: Advances in theory, measurement, and applied research (pp. 223-239). Washington, D.C.: American Psychological Asociation.

Carey, K. B., \& Correia, C. J. (1997). Drinking motives predict alcohol-related problems in college students. Journal of Studies on Alcohol and Drugs, 58, 100-105.

Carey, K. B., Scott-Sheldon, L. A. J., Carey, M. P., \& DeMartini, K. S. (2007). Individual-level interventions to reduce college student drinking: A meta-analytic review. Addictive Behaviors, 32, 2469-2492.

Cooper, M. L. (1994). Motivations for alcohol use among adolescents: Development and validation of a four-factor model. Psychological Assessment, 6, 117-128.

Cooper, M. L., Frone, M. R., Russell, M., \& Mudar, P. (1995). Drinking to regulate positive and negative emotions: A motivational model of alcohol use. Journal of Personality and Social Psychology, 69, 990-1005. 
Cooper, M. L., Russell, M., Skinner, J. B., Frone, M. R., \& Mudar, P. (1992). Stress and alcohol use: Moderating effects of gender, coping, and alcohol expectancies. Journal of Abnormal Psychology, 101, 139-152.

Courtney, K. E., \& Polich, J. (2009). Binge drinking in young adults: Data, definitions, and determinants. Psychological Bulletin, 135, 142-156.

Cox, W. M., \& Klinger, E. (1988). A motivational model of alcohol use. Journal of Abnormal Psychology, 97, 168-180.

Gazis, N., Connor, J. P., \& Ho, R. (2010). Cultural identity and peer influence as predictors of substance abuse among culturally diverse Australian adolescents. The Journal of Early Adolescence, 30, 345-368.

Gonzalez, V. M. (2012). Association of solitary binge drinking and suicidal behavior among emerging adult college students. Psychology of Addictive Behaviors, 26, 609-614.

Gonzalez, V. M., \& Skewes, M. C. (2013). Solitary heavy drinking, social relationships, and negative mood regulation in college drinkers. Addiction Research \& Theory, 21, 285-294.

Haines, M., \& Spear, S. F. (2010). Changing the perception of the norm: A strategy to decrease binge drinking among college students. American Journal of College Health, 45, 134140.

Hawkins, E. H. (2002). Navigating between two worlds: A sociocultural examination of alcohol problems among urban American Indian youth. (Unpublished doctoral dissertation). University of Washington, Seattle.

Hingson, R., Heeren, T., Winter, M., \& Weschler, H. (2002). Magnitude of alcohol-related mortality and morbidity among U.S. college students ages 18-24: Changes from 1998 to 2001. Annual Review of Public Health, 26, 259-279. 
Kassel, J. D., Jackson, S. I., \& Unrod, M. (2000). Generalized expectancies for negative mood regulation and problem drinking among college students. Journal of Studies on Alcohol and Drugs, 61, 332-340.

Knight, J. R., Wechsler, H., Kuo, M., Seibring, M., Weitzman, E. R., \& Schuckit, M. A. (2002). Alcohol abuse and dependence among U.S. college students. Journal of Studies on Alcohol and Drugs, 63, 263-270.

Kulis, S., Napoli, M., \& Marsiglia, F. F. (2002). Ethnic pride, biculturalism, and drug use norms of urban American Indian adolescents. Social Work Research, 26, 101-112.

Kuntsche, E., Knibbe, R., Gmel, G., \& Engels, R. (2005). Why do young people drink? A review of drinking motives. Clinical Psychology Review, 25, 841-861.

Kuntsche, E. (2007). Tell me...why do you drink? A study of drinking motives in adolescence. (Doctoral dissertation). Swiss Institute for the Prevention of Alcohol and Drug Problems.

Kuntsche, E., Stewart, S. H., \& Cooper, M. L. (2008). How stable is the motive-alcohol use link? A cross-national validation of the Drinking Motives Questionnaire Revised among adolescents from Switzerland, Canada, and the United States. Journal of Studies on Alcohol and Drugs, 69, 388-396.

LaBrie, J. W., Hummer, J. F., \& Pedersen, E. R. (2007). Reasons for drinking in the college student context: The differential role and risk of the social motivator. Journal of Studies on Alcohol and Drugs, 68, 393-398.

LaMarr, C. J. (2003). “Firewater myth:” Fact, fantasy, or self-fulfilling prophecy. (Unpublished doctoral dissertation). University of Washington, Seattle.

Mail, P. D. (2002). Multiple perspectives on alcohol and the American Indian. In P. D. Mail, S. Heurtin-Roberts, S. E. Martin, \& J. Howard (Eds.), Alcohol use among American 
Indians: Multiple perspectives on a complex problem (NIAAA Research Monograph No. 37; pp. 3-23). Bethesda, MD: National Institutes of Health, National Institute on Alcohol Abuse and Alcoholism.

Marsiglia, F. F., Kulis, S., \& Hecht, M. L. (2001). Ethnic labels and ethnic identity as predictors of drug use among middle school students in the Southwest. Journal of Research on Adolescence, 11, 21-48.

Marsiglia, F. F., Kulis, S., Hecht, M. L., \& Sills, S. (2004) Ethnicity and ethnic identity as predictors of drug norms and drug use among preadolescents in the US Southwest. Substance Use \& Misuse, 39, 1061-1094.

McNally, A. M., \& Palfai, T. P. (2001). Negative emotional expectancies and readiness to change among college student binge drinkers. Addictive Behaviors, 26, 263-270.

Neff, J. A. (1997). Solitary drinking, social isolation, and escape drinking motives as predictors of high quantity drinking among Anglo, African American, and Mexican American Males. Alcohol and Alcoholism, 32, 33-41.

Neighbors, C., Larimer, M. E., \& Lewis, M. A. (2004). Targeting misperceptions of descriptive drinking norms: Efficacy of a computer-delivered personalized normative feedback intervention. Journal of Consulting and Clinical Psychology, 72, 434-447.

O'Malley, P. M., \& Johnston, L. D. (2002). Epidemiology of alcohol and other drug use among American college students. Journal of Studies on Alcohol, 14, 23-39.

Oetting, E. R., Donnermeyer, J. F., Trimble, J. E., \& Beauvais, F. (1998). Primary socialization theory: Culture, ethnicity, and cultural identification. The links between culture and substance use. Substance Use \& Misuse, 33, 2075-2107.

Park, C. L. (2004). Positive and negative consequences of alcohol consumption in college 
students. Addictive Behaviors, 29, 311-321.

Perkins, H. W., \& Berkowitz, A. D. (1986). Perceiving the community norms of alcohol use among students: Some research implications for campus alcohol education programming. International Journal of Mental Health and Addiction, 21, 961-976.

Perkins, H. W., Haines, M. P., \& Rice, R. (2005). Misperceiving the college drinking norm and related problems: A nationwide study of exposure to prevention information, perceived norms, and student alcohol misuse. Journal of Studies on Alcohol and Drugs, 66, 470478.

Phinney, J. S., \& Ong, A. D. (2007). Conceptualization and measurement of ethnic identity: Current status and future directions. Journal of Counseling Psychology, 54, 271-281.

Pugh, L. A., \& Bry, B. H. (2007). The protective effects of ethnic identity for alcohol and marijuana use among Black young adults. Cultural Diversity and Ethnic Minority Psychology, 13, 187-193.

Read, J. P., Kahler, C. W., Strong, D. R., \& Colder, C. R. (2006). Development and preliminary validation of the Young Adult Alcohol Consequences Questionnaire. Journal of Studies on Alcohol, 67, 169-177.

Read, J. P., Wood, M. D., Kahler, C. W., Maddock, J. E., \& Palfai, T. P. (2003). Examining the role of drinking motives in college student alcohol use and problems. Psychology of Addictive Behaviors, 17, 13-23.

Saunders, J. B., Aasland, O. G., Babor, T. F., De La Fuente, J. R., \& Grant, M. (1993). Development of the Alcohol Use Disorders Identification Test (AUDIT): WHO collaborative project on early detection of persons with harmful alcohol consumption. Addiction, 88, 791-804. 
U.S. Department of Education, National Center for Education Statistics, Integrated Postsecondary Education Data System (2012, November). Fall 2001 and Spring 2007 through Spring 2012, Graduate Rates. Retrieved from http://nces.ed.gov/programs/digest/d12/tables/dt12 376.asp.

Wallace, J. M., Bachman, J. G., O’Malley, P. M., Johnston, L. D., Schulenberg, J. E., \& Cooper, S. M. (2002). Tobacco, alcohol, and illicit drug use: Racial and ethnic differences among U.S. high school seniors, 1976-2000. Public Health Reports, 117, S67-S75.

Wechsler, H., \& Nelson, T. F. (2001). Binge drinking and the American college student: What's five drinks? Psychology of Addictive Behaviors, 15, 721-734.

Whitesell, N. R., Beals, J., Big Crow, C., Mitchell, C. M., \& Novins, D. K. (2012). Epidemiology and etiology of substance use among American Indians and Alaska Natives: Risk, protection, and implications for prevention. The American Journal of Drug and Alcohol Abuse, 38, 376-382. 
Table 1. Means and standard deviations of scale scores for the study sample $(N=205)$.

\begin{tabular}{|l|l|l|l|l|}
\hline Variable & Sample Mean (SD) & Native Students & Non-Native Students & $t$ \\
& & Mean (SD) & Mean (SD) & \\
\hline DMQ Social & $12.71(.48)$ & $12.42(4.29)$ & $12.76(4.08)$ & .44 \\
\hline DMQ Coping & $10.01(3.96)$ & $9.88(4.20)$ & $10.04(3.93)$ & .21 \\
\hline DMQ Enhancement & $11.47(4.03)$ & $11.88(4.28)$ & $11.39(3.99)$ & -.62 \\
\hline DMQ Conformity & $6.94(2.83)$ & $7.09(2.96)$ & $6.91(2.81)$ & -.34 \\
\hline YAACQ & $11.98(10.67)$ & $16.30(11.46)$ & $11.15(10.34)$ & $-2.58^{*}$ \\
\hline AUDIT-C & $4.21(2.66)$ & $4.62(2.99)$ & $4.13(2.60)$ & -.97 \\
\hline BDI & $10.04(8.95)$ & $8.34(4.61)$ & $10.35(9.52)$ & 1.15 \\
\hline MEIM-EIS & $2.28(.70)$ & $2.80(.52)$ & $2.15(.65)$ & $-5.30^{* * *}$ \\
\hline MEIM-ABC & $2.95(.72)$ & $3.48(.53)$ & $2.83(.71)$ & $-4.88^{* * *}$ \\
\hline
\end{tabular}

Note. ${ }^{*} p<.05 ;{ }^{* * *} p<.001 ; t$ values for group mean differences between Native and non-Native students. 
Table 2. Regression model predicting past-year alcohol consequences controlling for frequency of binge drinking and social drinking motives.

\begin{tabular}{|l|l|l|l|}
\hline Predictor & Beta & $t$ & $95 \%$ CI \\
\hline Age & .03 & .51 & -.20 to .33 \\
\hline Gender & .08 & 1.31 & -.92 to 4.55 \\
\hline BDI & .24 & $3.91^{* * *}$ & .14 to .42 \\
\hline Binge episodes per month & .27 & $4.18^{* * *}$ & .48 to 1.34 \\
\hline DMQ-R Social & .23 & $3.50^{* *}$ & .26 to .91 \\
\hline Native vs. Non-Native & .28 & $4.07^{* * *}$ & 4.07 to 11.75 \\
\hline MEIM-EIS & .08 & 1.06 & -1.10 to 3.64 \\
\hline MEIM-ABC & -.25 & $-3.32^{* *}$ & -5.80 to -1.48 \\
\hline
\end{tabular}

Note. ${ }^{*} p<.05,{ }^{* *} p<.01,{ }^{* * *} p<.001$. Gender: female $=1$, male $=2$; Ethnicity: non-Native $=0$,

Native $=1$. Full model $R^{2}=.36 ; F(8,178)=12.70 ; p<.001$. Betas, $t$ values, and $95 \%$ confidence intervals for each regression coefficient listed are for the full model. 
Table 3. Regression model predicting past-year alcohol consequences controlling for frequency of binge drinking and coping motives.

\begin{tabular}{|l|l|l|l|}
\hline Predictor & Beta & $t$ & $95 \%$ CI \\
\hline Age & .03 & .58 & -.18 to .33 \\
\hline Gender & .05 & .74 & -1.67 to 3.65 \\
\hline BDI & .11 & 1.76 & -.02 to .28 \\
\hline Binge episodes per month & .27 & $4.32^{* * *}$ & .48 to 1.29 \\
\hline DMQ-R Coping & .35 & $5.21^{* * *}$ & .58 to 1.29 \\
\hline Native vs. Non-Native & .25 & $3.75^{* * *}$ & 3.34 to 10.77 \\
\hline MEIM-EIS & .10 & 1.35 & -.72 to 3.84 \\
\hline MEIM-ABC & -.24 & $-3.33^{* *}$ & -5.58 to -1.43 \\
\hline
\end{tabular}

Note. ${ }^{*} p<.05,{ }^{* *} p<.01,{ }^{* * *} p<.001$. Gender: female $=1$, male $=2$; Ethnicity: non-Native $=0$,

Native $=1$. Full model $R^{2}=.38 ; F(8,178)=15.44 ; p<.001$. Betas, $t$ values, and $95 \%$ confidence intervals for each regression coefficient listed are for the full model. 
Table 4. Regression model predicting past-year alcohol consequences controlling for frequency of binge drinking and enhancement motives.

\begin{tabular}{|l|l|l|l|}
\hline Predictor & Beta & $t$ & $95 \%$ CI \\
\hline Age & -.00 & -.02 & -.26 to .26 \\
\hline Gender & .06 & .94 & -1.47 to 4.17 \\
\hline BDI & .22 & $3.50^{* *}$ & .11 to .40 \\
\hline Binge episodes per month & .29 & $4.25^{* * *}$ & .51 to 1.40 \\
\hline DMQ-R Enhancement & .20 & $2.99^{* *}$ & .18 to .87 \\
\hline Native vs. Non-Native & .24 & $3.48^{* *}$ & 3.01 to 10.88 \\
\hline MEIM-EIS & .08 & 1.05 & -1.12 to 3.66 \\
\hline MEIM-ABC & -.25 & $-3.28^{* *}$ & -5.81 to -1.45 \\
\hline
\end{tabular}

Note. ${ }^{*} p<.05,{ }^{* *} p<.01,{ }^{* * *} p<.001$. Gender: female $=1$, male $=2$; Ethnicity: non-Native $=0$,

Native $=1$. Full model $R^{2}=.32 ; F(8,176)=12.04 ; p<.001$. Betas, $t$ values, and $95 \%$ confidence intervals for each regression coefficient listed are for the full model. 
Table 5. Regression model predicting past-year alcohol consequences controlling for frequency of binge drinking and conformity motives.

\begin{tabular}{|l|l|l|l|}
\hline Predictor & Beta & $t$ & $95 \%$ CI \\
\hline Age & -.00 & -.05 & -.27 to .26 \\
\hline Gender & .08 & 1.25 & -1.03 to 4.55 \\
\hline BDI & .24 & $3.79^{* * *}$ & .13 to .42 \\
\hline Binge episodes per month & .35 & $5.43^{* * *}$ & .75 to 1.60 \\
\hline DMQ-R Conformity & .12 & 1.92 & -.01 to .95 \\
\hline Native vs. Non-Native & .27 & $3.86^{* * *}$ & 3.80 to 11.75 \\
\hline MEIM-EIS & .11 & 1.34 & -.78 to 4.07 \\
\hline MEIM-ABC & -.26 & $-3.33^{* *}$ & -5.95 to -1.53 \\
\hline
\end{tabular}

Note. ${ }^{*} p<.05,{ }^{* *} p<.01,{ }^{* * *} p<.001$. Gender: female $=1$, male $=2$; Ethnicity: non-Native $=0$,

Native $=1$. Full model $R^{2}=.31 ; F(8,178)=11.34 ; p<.001$. Betas, $t$ values, and $95 \%$ confidence intervals for each regression coefficient listed are for the full model. 